\title{
Management of Bilateral Phlegmasia Cerulea Dolens in a Patient with Subacute Splenic Laceration
}

\author{
Hernan A Bazan, MD, ${ }^{1}$ Eric Reiner, DO, ${ }^{2}$ and Bauer Sumpio, MD, Ph.D ${ }^{1}$
}

Phlegmasia cerulea dolens (PCD) is a rare but limb-threatening complication of deep vein thrombosis. We report a case of a 76-year-old man with recent splenic trauma and inferior vena cava (IVC) filter placement, who developed bilateral lower extremity PCD. Utilizing an endoluminal approach, the patient underwent mechanical thrombectomy and thrombolysis through bilateral infusion catheters placed antegrade from bothpopliteal veins. Clot lysis and return of palpable pedal pulses occurred within 24 hours. We demonstrate that the endoluminal management of this disease may be cautiously applied to the trauma patient, and that the judicious use of thrombolytic therapy can be beneficial even in the patient with a high potential for hemorrhage.

Key words: phlegmasia, dolens, thrombolysis

\section{INTRODUCTION}

$\mathrm{O}$ ver 600,000 cases of venous thromboembolism are estimated to occur each year in the United States. ${ }^{1)}$ Although pulmonary embolism is a well-known complication of untreated acute deep venous thrombosis, a less frequent but serious manifestation of venous thrombosis are phlegmasia alba dolens (PAD), phlegmasia cerulea dolens (PCD), and venous gangrene. These form a clinical spectrum of the same disorder which are all limbthreatening and, potentially, life-threatening. We describe the endoluminal management of a patient with recent splenic trauma presenting with PCD and threatened lower limbs, demonstrating that even in the patient with recent solid organ trauma, the judicious use of thrombolysis may be of value.

${ }^{1}$ Departments of Surgery, Section of Vascular Surgery, and ${ }^{2}$ Diagnostic Radiology, Yale University School of Medicine, New Haven, CT, USA

Received: April 5, 2007 Accepted: September 28, 2007 Address for reprint requests to Hernan A Bazan, MD: 533 Bolivar St, Room 524, Department of Surgery, Section of Vascular Surgery, Louisiana State University Health Sciences Center, New Orleans, LA 70112, USA

\section{CASE}

A 76-year-old man without any significant medical history fell from a ladder while tending to house work a month prior and sustained a grade III splenic laceration; he was treated non-operatively with bed rest and discharged home. He returned to our hospital three weeks later with signs and symptoms consistent with a pulmonary embolus, which was confirmed with a computed tomography (CT) scan angiogram of the chest. A TrapEase ${ }^{\circledR}$ (Cordis, Miami, FL) inferior vena cava (IVC) was placed, as it was deemed that he would be at high-risk for an intra-abdominal bleed and, therefore, could not be anticoagulated given the recent splenic laceration. Following this intervention, he returned a few days later to an outside institution with severe bilateral leg swelling, lower extremity pain, paresthesias, and non-palpable pedal pulses. There was no loss of motor function. He was transferred to our institution and found to have threatened limbs, and a diagnosis of PCD was made. During the one hour ambulance ride, he required seven liters of crystalloid fluid resuscitation due to the hypovolemic state from the poor venous return, secondary to large amounts of subcutaneous fluid sequestration.

He was promptly brought to the interventional radiology suite, placed in a prone position, and infusion catheters were placed antegrade, from both popliteal veins. 

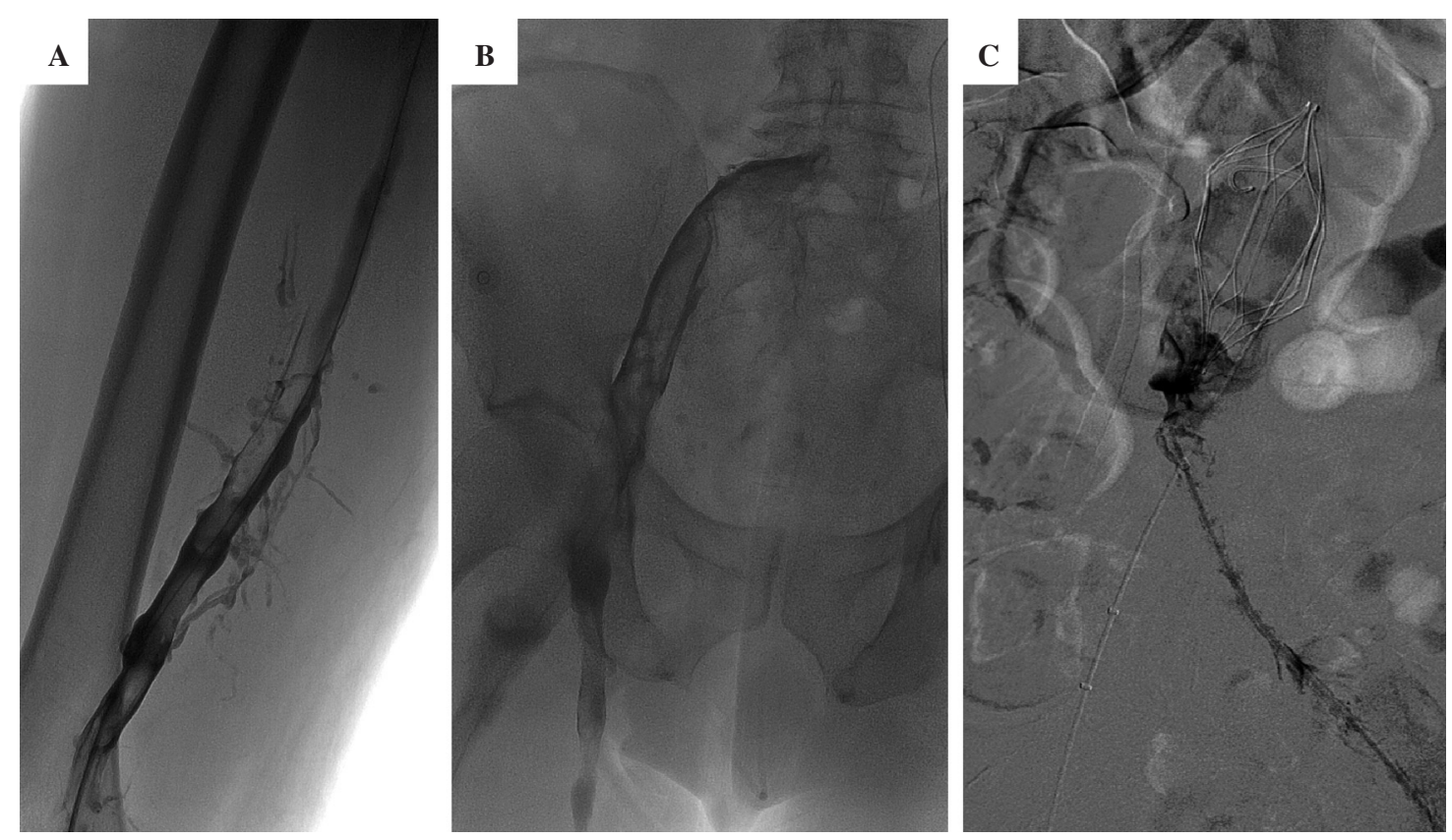

Fig. 1 Extensive thrombus from the inferior vena cava (IVC) to the popliteal vein.

A: Femoropopliteal venogram at day \#0 reveals extensive thrombus.

B: Iliofemoral venogram at day \#0.

C: Venogram at day \#1 demonstrates extensive amounts of caval thrombus.

Note the IVC filter. An AngioJet ${ }^{\circledR}$ mechanical thrombectomy catheter (Possis Medical, Minneapolis, MN) was used to help clear the clot burden in the IVC.

Thrombolysis was initiated employing power pulse recombinant tissue plasminogen activator (rTPA, $4 \mathrm{mg}$ ) and subsequent slow rTPA infusion $(0.5 \mathrm{mg} /$ hour $)$, followed by low-doses of anticoagulation (400 units/hour through each side-arm sheath). The initial venogram demonstrated extensive thrombosis of the femoral and iliac venous systems (Fig. 1A and B). A substantial amount of clot was removed from the veins. The patient was placed in bed rest with elevation of his lower extremities. Within a couple of hours, the extremities became softer and the patient's pain and paresthesias resolved. On post-procedure day \#1, there was a marked improvement in the patency of the femoral and external iliac veins, with a return of palpable pedal pulses. However, some non-occlusive clot remained in the popliteal and iliac veins. Furthermore, there was only minimal resolution of thrombus around the TrapEase ${ }^{\circledR}$ IVC filter (Fig. 1C). An AngioJet ${ }^{\circledR}$ mechanical thrombectomy catheter (Possis Medical, Minneapolis, MN) was advanced into the vena cava just caudal to the IVC filter, in the location of the extensive thrombus, and mechanical thrombolysis was performed subjacent to the IVC filter. By post-procedure day \#2 (Fig. 2A), there was resolution of thrombus in the iliac veins, but persistent clot in the IVC so thrombolysis was continued. Anticoagulation was increased to 500 units/ hour through each sheath. A venogram on post-procedure day \#3 (Fig. 2B), demonstrated some residual clot around the IVC filter. An interim CT scan of the abdomen and pelvis demonstrated a stable splenic hematoma (Fig. 3A) and the IVC was found to be well-distended above its normal size relative to the aorta (Fig. 3B). A venogram on post-procedure day \#4 demonstrated improvement in the residual intra-filter thrombus (Fig. 2C) and the rTPA treatment was discontinued. The patient was then fully anticoagulated with heparin and transitioned over to warfarin therapy with a target international normalized ratio of 2-3 and discharged home. He has done well through 6 months of follow-up.

\section{DiscuSSION}

Phlegmasia is a rare limb-threatening manifestation of massive venous thrombosis and obstruction of the venous drainage of an extremity. The patient with severe deep venous thrombosis presents with threatened limbs and requires prompt evaluation and therapy. PAD progresses to PCD when the collateral venous channels thrombose, transforming a blanched state (white or alba) to cyanosis 

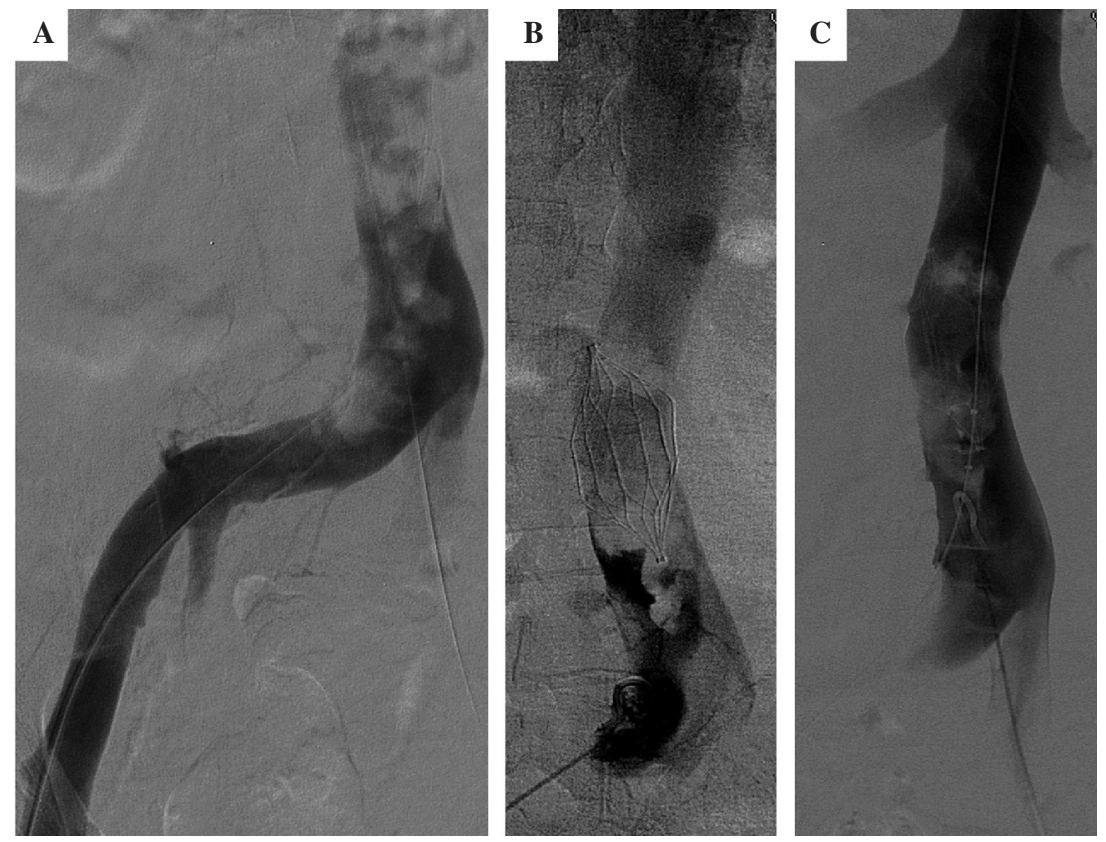

Fig. 2 Improvement in the thrombus burden after pharmachomechanical thrombolysis. A: At day \#2, there is still iliac vein thrombus

B: Caval thrombus persists on day \#3 C: By thrombolysis day \#4, smaller amounts of intra-filter thrombus is present; thrombolysis was discontinued
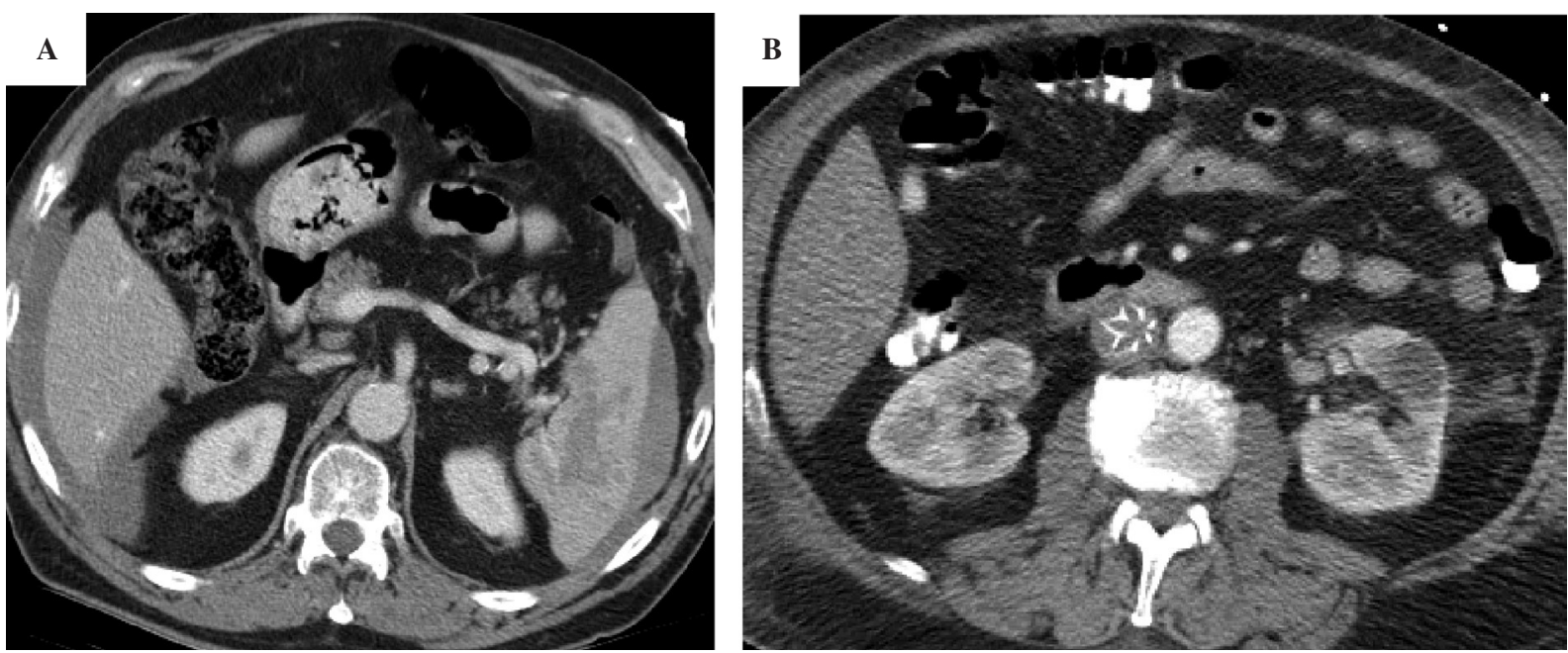

Fig. 3 Axial CT scan of the abdomen was done to evaluate known peri-splenic hematoma.

A: Stable peri-hepatic and peri-splenic fluid

B: Caval thrombus around the IVC filter causing engorgement of the IVC

(blue or cerulea). In both PAD and PCD, there is massive superficial and deep venous thrombosis; however, in PAD there is patency of the venous collaterals so that it rarely results in limb loss. In contrast, in PCD, the acute and complete venous thrombosis results in arterial compromise, systemic hypovolemic shock from large amounts of fluid sequestration and, left untreated, venous gangrene. There is wide consensus that treatment of patients with PCD should consist of timely intervention; however, these interventions are not without risk, including a potential for hemorrhagic stroke, especially in the elderly patient. In this particular case, a repeat bleed from the spleen could constitute the indication for an emergent splenectomy. Alternatively, if the patient was relatively hemodynamically stable, an attempt at coil and gelfoam embolization of the bleeding splenic segment could be attempted. Realizing these potential complications, we demonstrate that the judicious use of thrombolytic therapy can be beneficial even in the patient presenting with recent trauma and confounding solid organ trauma. 
Color Doppler and gray-scale ultrasound is the imaging modality of choice to substantiate the diagnosis of PCD. There is no need for magnetic resonance venous imaging or conventional venography to make the diagnosis. Sonography has been shown to have $95 \%$ sensitivity and $98 \%$ specificity in diagnosis DVT, making sonography a highly effective and efficient means of diagnosing venous thrombi in patients with phlegmasia. ${ }^{2)}$ Once the diagnosis is made and the patient's resuscitation begins, however, endovenous imaging and treatment play the major role in its management.

Malignancy is the most common cause of non-traumatic venous thrombosis and accounts for approximately $20-40 \%$ of the patients with PCD. Other etiologic factors of severe venous thrombosis include a hypercoaguable syndrome (factor V Leiden mutation, antithrombin deficiency, protein C or S deficiency, prothrombin $20210 \mathrm{mu}-$ tation, or activated protein $\mathrm{C}$ resistance), the presence of a vena cava filter, recent trauma, ulcerative colitis, heart failure, mitral valve stenosis, and compression of the left common iliac vein by the right iliac common artery (May-Thurner syndrome). ${ }^{3)}$ Pregnancy may also rarely be associated with phlegmasia, especially during the third trimester when the uterus is large enough to compress the left common iliac vein against the pelvic rim (milk leg syndrome). The exact incidence of these rare disorders is not well established in the literature. PCD may occur at any age but is more common during the fifth and sixth decades of life, likely due to an increased incidence of etiologic factors (such as malignancy); its incidence is thought to be higher in females than males. Another facet of the clinical presentation of PCD may be hypovolemic shock, as in this patient. The massive fluid sequestration may lead to bleb and bullae formation.

If phlegmasia is left untreated, venous thrombosis can extend to involve the capillaries, which it does in 40-60\% of cases of PCD, and ischemia occurs causing an irreversible venous gangrene involving the skin, subcutaneous tissue, and muscle. When the venous gangrene is superficial, arterial pulses may still be palpable; however, gangrene involving the muscular compartment may result in increased compartmental pressures and a pulse deficit. The role of arterial spasm in this process is not well-delineated. The pulses, though, are likely very difficult to appreciate due to the significant edema and for this reason an ankle-brachial index (ABI) should be done to document the patient's arterial examination. In a patient without any significant risk factors for peripheral obstructive vascular disease, an ABI less than normal should be considered alarming in this setting.

A multicenter venous registry for catheter-directed thrombolysis supports the use of catheter-directed thrombolysis. ${ }^{4)}$ Data collected on almost 500 patients demonstrated significant venous lysis with catheter-directed thrombolysis ( $>50 \%)$ in $80 \%$ of patients, and complete venous lysis in one-third. The authors reported increased success with iliofemoral compared to femoropopliteal thrombosis and in patients presenting acutely (less than 10 days). Major complications such as pulmonary embolism $(1.2 \%)$ and mortality $(0.4 \%)$ were rare. Since anticoagulation is a basic part of the treatment of PCD, in cases of heparin-induced thrombocytopenia lepirudin, a direct thrombin inhibitor, should be used.5) To help circumvent the venous hypertension and prevent the post-phlebitic syndrome, the patient should be instructed to wear longterm prescription compression stockings with at least $30-40 \mathrm{mmHg}$ of graded pressure.6)

PAD, PCD and venous gangrene remain a challenge for the vascular surgeon. Treatment modalities continue to evolve. We demonstrate that the judicious use of thrombolytic therapy can be beneficial even in the patient with PCD and a high potential for bleed, such as a patient with recent trauma.

\section{REFERENCES}

1) Silverstein MD, Heit JA, Mohr DN, Petterson TM, O'Fallon WM, Melton LJ 3rd. Trends in the incidence of deep vein thrombosis and pulmonary embolism: a 25-year population-based study. Arch Intern Med. 1998; 158: 585-93.

2) Fraser JD, Anderson DR. Deep venous thrombosis: recent advances and optimal investigation with US. Radiology. 1999; 211: 9-24.

3) Rutherford RB, Johnston W. In: Cronenwett JL, et al eds. Vascular Surgery Online, 6th Edition. Philadelphia: W.B. Saunders, 2005.

4) Mewissen MW, Seabrook GR, Meissner MH, Cynamon J, Labropoulos N, Haughton SH. Catheterdirected thrombolysis for lower extremity deep venous thrombosis: report of a national multicenter registry. Radiology. 1999; 211: 39-49.

5) Mousa A, Henderson P, Dayal R, Bernheim J, Kent KC, Faries PL. Endoluminal recanalization in a patient with phlegmasia cerulea dolens using a multimodality approach. Vascular. 2005; 13: 313-7.

6) Coburn M, Ashworth C, Francis W, Morin C, Broukhim M, Carney WI Jr. Venous stasis complications of the use of the superficial femoral and popliteal veins for lower extremity bypass. J Vasc Surg. 1993; 17: 1005-8. 